\title{
Number of discernible colors for color-deficient observers estimated from the MacAdam limits
}

\author{
Esther Perales, ${ }^{1, *}$ Francisco Miguel Martínez-Verdú, ${ }^{1}$ João Manuel Maciel Linhares, ${ }^{2}$ and \\ Sérgio Miguel Cardoso Nascimento ${ }^{2}$ \\ ${ }^{1}$ Department of Optics, University of Alicante, Carretera de San Vicente del Raspeig s/n 03690, Alicante Spain \\ ${ }^{2}$ Department of Physics, Campus de Gualtar, University of Minho, 4710-057 Braga, Portugal \\ *Corresponding author: esther.perales@ua.es
}

Received December 23, 2009; revised July 26, 2010; accepted July 30, 2010;

posted August 3, 2010 (Doc. ID 121965); published September 2, 2010

\begin{abstract}
We estimated the number of colors perceived by color normal and color-deficient observers when looking at the theoretic limits of object-color stimuli. These limits, the optimal color stimuli, were computed for a color normal observer and CIE standard illuminant D65, and the resultant colors were expressed in the CIELAB and DIN99d color spaces. The corresponding color volumes for abnormal color vision were computed using models simulating for normal trichromatic observers the appearance for dichromats and anomalous trichomats. The number of colors perceived in each case was then computed from the color volumes enclosed by the optimal colors also known as MacAdam limits. It was estimated that dichromats perceive less than $1 \%$ of the colors perceived by normal trichromats and that anomalous trichromats perceive 50\%-60\% for anomalies in the medium-wavelength-sensitive and $60 \%-70 \%$ for anomalies in the long-wavelength-sensitive cones. Complementary estimates obtained similarly for the spectral locus of monochromatic stimuli suggest less impairment for color-deficient observers, a fact that is explained by the two-dimensional nature of the locus. (C) 2010 Optical Society of America
\end{abstract}

OCIS codes: $330.1730,330.1690,330.1720,330.6180$.

\section{INTRODUCTION}

Normal color vision is determined by three cone photopigments with spectral sensitivity maxima in the long-, medium-, and short-wavelength regions of the visible spectrum [1,2]. This trichromatic sampling of the spectrum forms the basis for discrimination of a large number of colors in natural environments. Estimates of the number of discernible colors for normal color vision have been obtained from discrimination data [3], from the theoretical limits of natural colors [4-8], and from the analysis of empirical data sets of hyperspectral data of natural scenes [9-11]. Numbers vary according to the methodology and assumptions of the analysis, but a number between one and two million seems to include more comprehensive estimates from the theoretical limits [6,7] and from the natural scenes $[9,10]$.

Abnormal color vision affects a considerable fraction of the population and arises from congenital or acquired deficiencies that impose limitations on color discrimination. $\mathrm{X}$-linked deficiencies affect almost $10 \%$ of males $[1,12,13]$. Red-green anomalous trichromats, protanomals or deuteranomals, have the long- and the medium-wavelengthsensitive pigments respectively, spectrally shifted in relation to normal and represent about $6 \%-7 \%$ of the male population. Protanopes lack the long-wavelengthsensitive pigment and deuteranopes lack the mediumwavelength-sensitive pigment, and jointly represent about $2 \%$ of the male population. Hereditary anomalies in the short-wavelength-sensitive pigment are not sex linked and are less frequent: tritanopes, missing the short-wavelength-sensitive pigment, represent less than
$0.001 \%$ of the population, and it is not certain that tritanomals exist as they have never been well documented. Acquired color vision deficiencies will not be considered here $[14,15]$. Although there may be some advantages of color vision deficiencies $[16,17]$, an immediate effect is the reduction in chromatic discrimination, hence a reduction in the number of perceived colors. However, how much this impairment affects the number of colors perceived is still an open question.

Quantitative estimates of the reduction in chromatic diversity perceived by dichromats were obtained for natural scenes from the analysis of hyperspectral data and using models that simulate for normal trichromatic observers the appearance for color abnormal [10]. The results suggest that in natural scenes dichromats can perceive only about $7 \%$ of the colors normal observers can distinguish. Estimates for anomalous observers indicate an impairment of $60 \%-70 \%$ for natural scenes [18] and with a specific sample of artistic paintings an impairment in the range $78 \%-70 \%$ [19].

These estimates were based on the analysis of a partial volume of the color space, as only a fraction of the possible colors were actually found in nature [9]. Estimates based on the theoretical limits are independent of the experimental methodology and sampling and can provide firm complementary information. The theoretical limits are the optimal colors that enclose the object-color solid representing all colors arising only by reflection (or transmission) [20]. The theory underlining the spectral properties of optimal colors was developed early in the 20th century [21]; the corresponding loci were computed in a color dia- 
gram [22,23] and were later recalculated by MacAdam [24,25] to obtain what are known as the MacAdam limits or Rösch-MacAdam limits.

To estimate the number of discernible colors in complex stimuli as in natural scenes is necessarily an approximate exercise given the nature of the assumptions and simplifications that need to be considered. This applies even more strongly to color-deficient vision because of the limitations of the perceptual models available. Yet, the quest for quantification of chromatic discrimination as number of discernible colors has stimulated distinguished color scientists such as von Kries [26], MacAdam [3], Judd [27], and Krauskopf [28], among several others. This probably reflects the attractiveness of simplifying the very complex phenomenon of color discrimination but also has several practical applications that have been explored more recently in display gamut research $[7,29]$, colored filters [10], and color rendering $[4,5,30]$. For abnormal color vision the generalizations of these practical applications is of natural interest. Additionally, being able to quantify, even approximately, how much chromatic discrimination is impaired for each deficiency is of great interest for a simple straightforward description of deficiencies.

The aim of this work was to quantify how much chromatic diversity perceived by color-deficient observers is reduced when looking at the set of all object-color stimuli, that is, all physically possible illuminated surfaces arising only by reflection (or transmission). The optimal color stimuli for the normal observer were computed for the CIE standard illuminant D65 and expressed in the CIELAB [31] and DIN99d [32] color spaces. The corresponding color volumes for abnormal color vision were computed using models simulating for normal trichromatic observers the appearance for dichromats and anomalous trichromats. The number of colors perceived by each class of observers was then estimated by the color volumes enclosed by the MacAdam limits. In order to compare the level of impairment obtained for color volumes with that obtained for color surfaces, the number of colors perceived across the color surface defining the MacAdam limits and across the color surface corresponding to the spectral locus of monochromatic stimuli was also estimated.

\section{METHODS}

\section{A. Optimal Colors}

1. Color Normal

The optimal colors for the normal observer under standard illuminant D65 were obtained following MartínezVerdú et al. [4]. The spectral range of the reflectance functions was from 380 to $780 \mathrm{~nm}$ with a spectral sampling of $0.1 \mathrm{~nm}$. The lightness planes computed were in the range 1-99 in unitary steps. The $X Y Z$ tristimulus values were computed using the CIE 1931 color-matching functions [31] linearly interpolated for $0.1 \mathrm{~nm}$, and their representation in the CIELAB [31] and DIN99d [32] color spaces was also calculated. The CIELAB space was selected for comparison with previous estimates and DIN99d was selected because of its improved uniformity over CIELAB but still has attributes similar to those of the CIELAB formula [32,33].

\section{Dichromats}

For dichromats, a computational algorithm simulating for normal trichromatic observers the appearance of the optimal colors for the dichromatic observer [34,35] was applied. The method is based on assumptions concerning the hues that appear the same to dichromats and normal trichromats and rely on reports of unilateral inherited color vision [36]. This algorithm represents the color stimuli in a three-dimensional $(L, M, S)$ space and replaces each stimulus by its projection $\left(L^{\prime}, M^{\prime}, S^{\prime}\right)$ onto a reduced stimulus surface defined by a neutral axis and by the LMS locations of those monochromatic stimuli that are perceived as the same by normal trichromats and a given type of dichromat [36]. Here, Smith and Pokorny's fundamentals [37] were used to compute cone excitations. This set of fundamentals was based on a transformation of Judd's modification of the color matching functions of the $1931 \mathrm{CIE}$ standard observer. Thus, the optimal tristimulus values $(X, Y, Z)$ derived for the normal observers as explained above first had to be converted to $\left(X_{J u d d}, Y_{J u d d}, Z_{J u d d}\right)$, which was done with the help of Vos's conversion formula [38] and the necessary assumption that Judd's modified photopic luminous efficiency function $\mathrm{Y}_{\text {Judd }}$ coincided with the non-modified function, that is, $\mathrm{Y} \approx \mathrm{Y}_{\text {Judd }}$. Because Judd's modified function affects only the spectral region below $460 \mathrm{~nm}$ (see [20], p. 330 ), it is unlikely that this approximation has a considerable effect on the estimates obtained here. The $(L, M, S)$ excitations were then obtained for Smith and Pokorny's fundamentals [39] as defined by Wyszecki and Stiles [20]. The new $\left(L^{\prime}, M^{\prime}, S^{\prime}\right)$ were converted back into $\left(X^{\prime}, Y^{\prime}, Z^{\prime}\right)$ using the inverse transformations, and the new representations in CIELAB and DIN99d were computed. Notice that with this computational method what was computed was the color volume defining the optimal colors for normal observers as seen by dichromats.

\section{Anomalous Trichromats}

For anomalous trichromats, anomalous cone spectral sensitivities were used. To simulate the average anomalous observer, the DeMarco et al. data were used [39,40]. For the protanomals, the $\mathrm{M}$ and $\mathrm{L}^{\prime}$ cone pigments are separated by $10 \mathrm{~nm}$; and for the deuteranomals, the $\mathrm{M}^{\prime}$ and $\mathrm{L}$ cone pigments are separated by $6 \mathrm{~nm}$. These sensitivities rely on the assumptions that anomalous observers can be characterized by average photopigments, that normal and anomalous trichromats have similar ocular media and optical densities of cone photopigments, and that photopigment spectra are relatively shape invariant when plotted as a function of the frequency. To get an estimate of the extent of the individual differences among anomalous trichromats, anomalous cone spectral sensitivities corresponding to larger and smaller spectral separations between $\mathrm{M}$ and $\mathrm{L}^{\prime}$ and $\mathrm{M}^{\prime}$ and $\mathrm{L}$ cone photopigments were estimated from sets of sensitivities derived from the wavelengths of the maxima absorption spectra of redgreen and green-red hybrid pigment spectra obtained by photobleaching difference absorption spectroscopy [41]. The computational procedure used to obtain these sensitivities consisted of computing the absorbance spectrum from the cone fundamentals, spectrally shifting the appropriate amount in a frequency scale in each case, and 
then reconstructing the new fundamentals. For protanomals, the $\mathrm{M}$ and $\mathrm{L}^{\prime}$ cone pigments' maximum and minimum spectral separations were $11 \mathrm{~nm}$ and $4 \mathrm{~nm}$, respectively, and for deuteranomals, the $\mathrm{M}^{\prime}$ and $\mathrm{L}$ maximum and minimum spectral separations were 11 and $2 \mathrm{~nm}$, respectively. Hereafter, references to anomalous trichromats concern the average anomalous observer of De Marco et al.

These cone spectral sensitivities were used to simulate the color perception for anomalous trichromats of the optimal reflectances set. Smith and Pokorny's transformation [37] was used to convert anomalous cone excitations into XYZ tristimulus values of Judd Vos's transformation [38] was then applied to transform the data into CIE 1931 $\mathrm{XYZ}$, and the representation in CIELAB and DIN99d color spaces was computed.

\section{B. Spectrum Locus of Monochromatic Stimuli}

The spectrum locus of monochromatic stimuli was computed in the CIELAB and DIN99d color spaces for constant lightness profiles from $L^{*}=0$ to $L^{*}=100$ with a step of $\Delta L^{*}=1$. The spectral range was from 380 to $780 \mathrm{~nm}$ with a spectral sampling of $0.1 \mathrm{~nm}$. The illuminant D65 was used as reference illuminant and the CIE 1931 standard observer was used to compute the XYZ tristimulus, which were then transformed into the CIELAB and DIN99d color spaces. For the anomalous observers, the cone anomalous spectral sensitivities were used. For dichromats, the procedure was as for optimal colors: the $(L, M, S)$ excitations were replaced by the projections $\left(L^{\prime}, M^{\prime}, S^{\prime}\right)$ according to Brettel's algorithm [34] and the new excitations converted back into $\left(X^{\prime}, Y^{\prime}, Z^{\prime}\right)$ and represented in CIELAB and DIN99d color spaces.

\section{Number of Discernible Colors}

For the Rösch-MacAdam color solid the number of discernible colors was estimated for each class of observers as follows. A square packing algorithm [42] was used. It consisted of computing the partial counts of discernible colors for each constant lightness plane. For CIELAB, the squares had a unit area corresponding to a discrimination threshold of 1 unit, and for DIN99d the squares had an area of 0.36 corresponding to a threshold of 0.6 (see Appendix A for the justification of this threshold). In each constant lightness plane, the algorithm draws the first square around the achromatic point and non-overlapping squares are drawn in sequence. In this way, the sum of these partial counts from $L^{*}=1$ to 99 , in steps of 1 or 0.6 for CIELAB and DIN99d, respectively, gives an estimate of the total number of discernible colors. This methodology was selected because it is only moderately demanding in computational power; on the other hand, what is important in the present work is that the numbers are relative rather than absolute, and it is expected that the underlying approximations of this methodology have only minor implications. For the dichromatic observers, the surface defined by the optimal colors in each constant lightness plane is converted into a line; therefore the algorithm counts the number of non-empty squares along that line.

For the Rösch-MacAdam color surface and for the spectrum locus of monochromatic stimuli the number of dis- cernible colors was estimated by counting the number of squares (with the unit area for CIELAB and 0.36 for DIN99d) along the line defined by the optimal colors and monochromatic stimuli, respectively. Results for the complete surfaces were obtained by summing across the levels of lightness.

\section{RESULTS}

Figure 1 shows a representation in the CIELAB color space of the color solid under the illuminant D65 as perceived by normal, dichromat, and anomalous observers. Figure 2 shows similar data for the DIN99d color space. For the CIELAB representation, the color volume for anomalous observers is a little smaller than the color volume for the normal observers. For dichromatic observers, the color solid is transformed into a surface with a specific orientation for each type of dichromacy: for the tritanope observer the surface is aligned along a red-green line and for the protanope and deuteranope along a blue-yellow line. The DIN99d representation shows similar features with a reduction in the volume that is somewhat smaller than in CIELAB. For the dichromatic observers, the color solid is also transformed into a surface, although more curved for the deuteranopic and protanopic observers in particular.

For better visualization of the differences between normal and deficient observers, Fig. 3 represents only the lightness plane of $L^{*}=50$ in both the CIELAB and the DIN99d color spaces. The main reduction for anomalous observers is found for the red and green regions of the color space. In the DIN99d color space the blue and yellow regions remain almost unchanged, but for the CIELAB color space the blue region changed a little. This difference between the representations in the two color spaces may be due to the reported non-uniformity of the CIELAB space in the blue region [43]. The analysis of the graphs also shows that there are colors for the anomalous observers that the normal observer cannot perceive, that is, colors with no perceptual correspondence. The effect, however, is small and color space dependent. For dichromatic observers, the surface defined by the MacAdam limits is converted into a line that is very similar to that for deuteranopic and protanopic observers.

Table 1 shows the estimates for the number of discernible colors obtained for the Rösch-MacAdam color solid and for the Rösch-MacAdam color surface expressed in the CIELAB color space for normal and color-deficient observers. Table 2 represents similar estimates obtained with the DIN99d color space. For the normal observers and CIELAB the value is about 2.3 million, a number close to the 2.28 obtained by Pointer and Attridge [7] and 2.22 obtained by Linhares et al. [9]. The number of colors obtained in DIN99d was similar, around 2 million. The impairments obtained for color-deficient observers are similar in the two spaces, with a tendency to be slightly larger in CIELAB. Protanomals perceive more colors than deuteranomals, a difference that can be explained by the spectral separation of the long- and medium-wavelengthsensitive photopigments, which is $10 \mathrm{~nm}$ for protanomals and $6 \mathrm{~nm}$ for deuteranomals. The three types of dichro- 

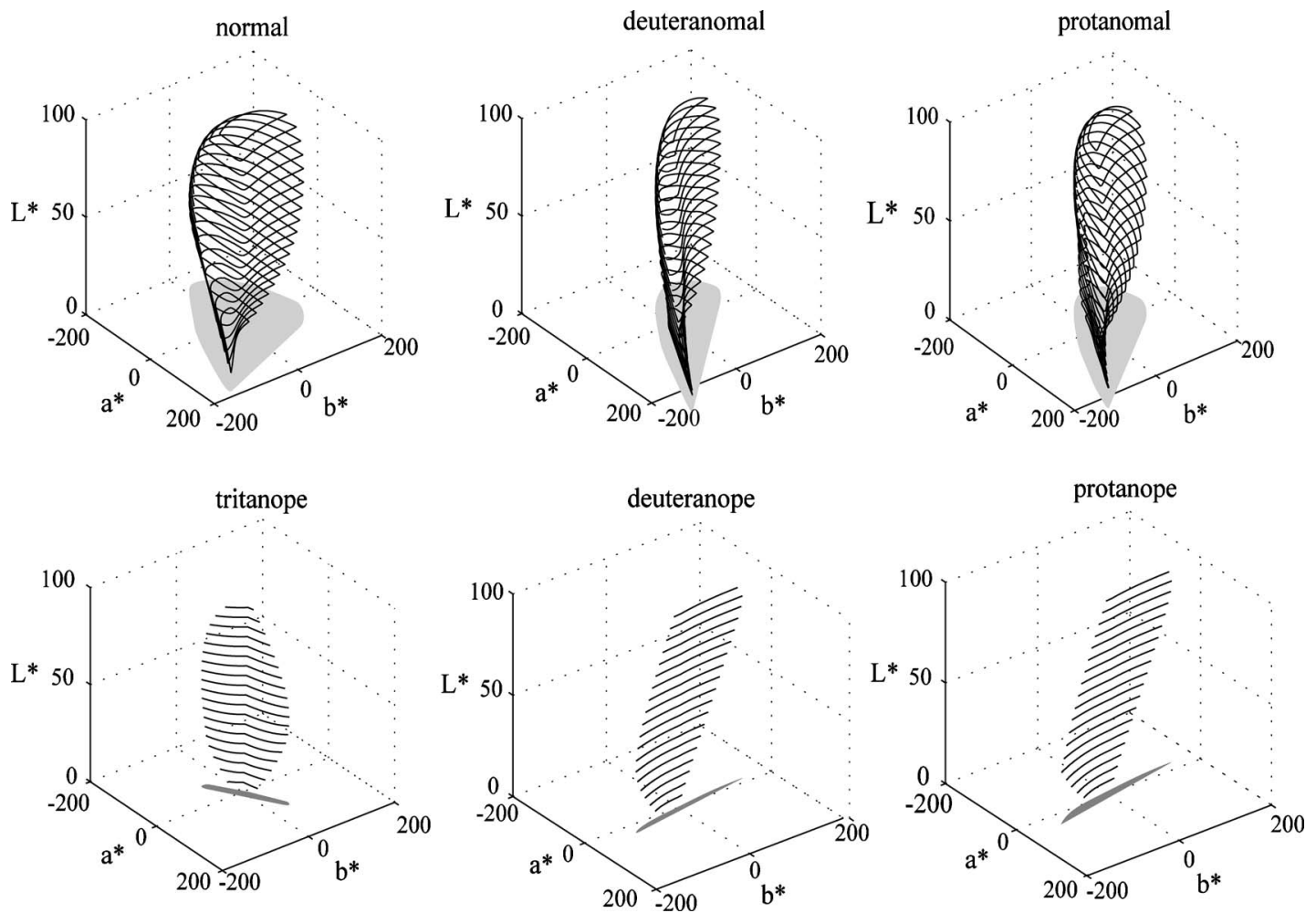

Fig. 1. Rösch-MacAdam color solid in the CIELAB color space under illuminant D65 for normal and color-deficient observers.

matic observers perceive about the same number of colors, less than $1 \%$ of the colors perceived by the normal trichromat.

For protanomals, estimates obtained in the CIELAB color space for the larger and smaller spectral difference in $\mathrm{M}$ and $\mathrm{L}^{\prime}$ (see Section 2, Methods, for details) gave an increase of $20 \%$ and a decrease of $30 \%$, respectively, in the number of colors perceived by the average anomalous observer of De Marco et al. For deuteranomal estimates, the larger and smaller spectral difference in $\mathrm{M}$ and $\mathrm{L}^{\prime}$ gave an increase of $35 \%$ and a decrease of $40 \%$, respectively, in the number of colors perceived by the average anomalous
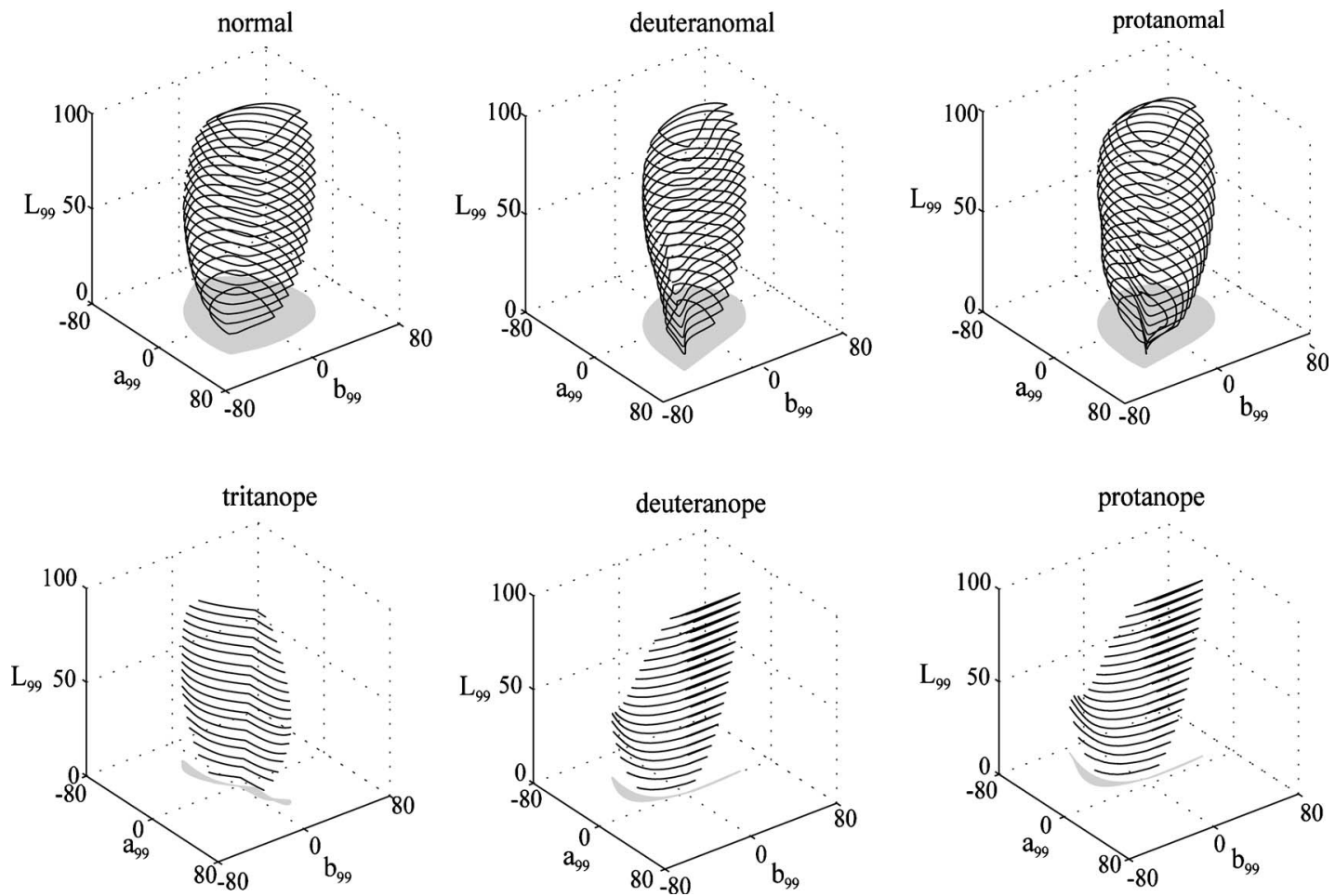

Fig. 2. Rösch-MacAdam color solid in the DIN99d color space under illuminant D65 for normal and color-deficient observers. 

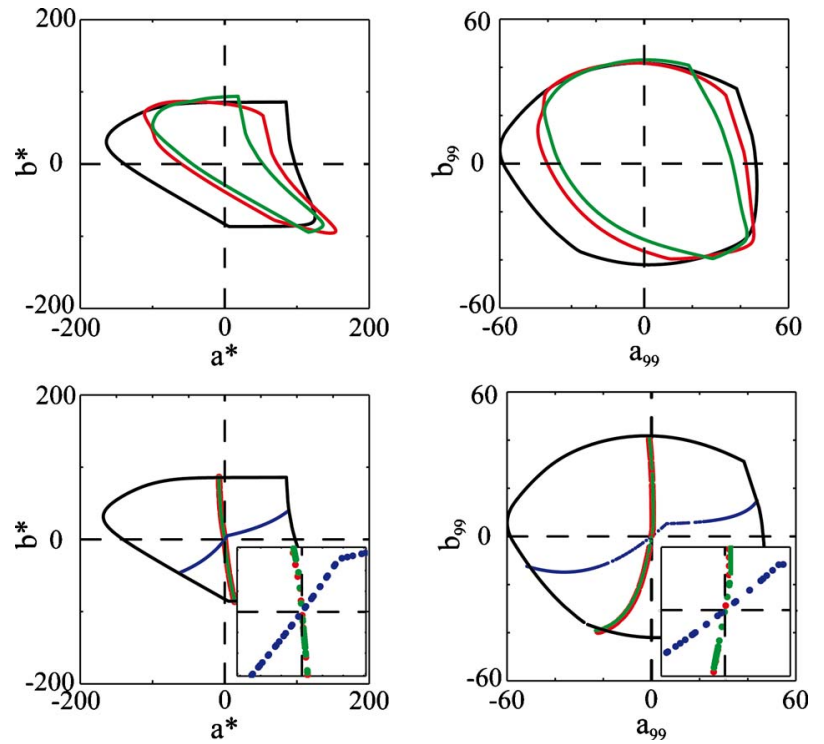

Fig. 3. (Color online) Comparison between the MacAdam limits for the constant lightness plane $\mathrm{L}^{*}=50$ associated with normal and color-deficient observers in the CIELAB (left) and DIN99d (right) color spaces. Top: normal observer [black (outer) line]), protanomal [red (middle) line], and deuteranomal observer [green (inner) line]. Bottom: tritanope [blue line (across center) and blue dots (diagonal)], deuteranope (green line and green dots), and protanope (red line and red dots). The red and green lines and dots are almost exactly superimposed. The insets represent amplifications of the central part of the corresponding graphs.

observer of De Marco et al. Estimates obtained with DIN99d were similar to those obtained with CIELAB.

Figure 4 shows a representation in the CIELAB color space of the spectral locus of the monochromatic stimuli as perceived by normal, dicromatic, and anomalous observers. Figure 5 shows similar data for the DIN99d color space. In both figures the spectral locus is an open surface because the points corresponding to the spectral extremes were not connected. Table 3 shows the estimates for the number of discernible colors in the spectral locus of monochromatic stimuli expressed in the CIELAB and DIN99d color spaces for normal and color-deficient observers. As expected, the level of impairment for the anomalous trichromats and dichromats is much smaller than the one

Table 1. Number of Discernible Colors Estimated for the Rösch-MacAdam Color Solid and for the Rösch-MacAdam Color Surface Expressed in the CIELAB Color Space for Normal and Color-Deficient Observers

\begin{tabular}{|c|c|c|}
\hline \multicolumn{3}{|c|}{ Number of Discernible Colors (D65) CIELAB } \\
\hline & $\begin{array}{c}\text { Rösch-MacAdam } \\
\text { Color Solid }\end{array}$ & $\begin{array}{c}\text { Rösch-MacAdam } \\
\text { Color Surface }\end{array}$ \\
\hline Normal & $2,341,419$ & 74,080 \\
\hline Protanomal & $1,455,539(62 \%)$ & $77,553(104 \%)$ \\
\hline Deuteranomal & $1,151,879(49 \%)$ & $76,800(103 \%)$ \\
\hline Tritanope & $17,695(0.76 \%)$ & $17,612(23 \%)$ \\
\hline Deuteranope & $17,185(0.73 \%)$ & $16,790(22 \%)$ \\
\hline Protanope & $18,052(0.77 \%)$ & $17,783(24 \%)$ \\
\hline
\end{tabular}

Table 2. Number of Discernible Colors Estimated for the Rösch-MacAdam Color Solid and for the Rösch-MacAdam Color Surface Expressed in the DIN99d Color Space for Normal and Color-Deficient Observers

\begin{tabular}{|c|c|c|}
\hline \multicolumn{3}{|c|}{ Number of Discernible Colors (D65) DIN99d } \\
\hline & $\begin{array}{c}\text { Rösch-MacAdam } \\
\text { Color Solid }\end{array}$ & $\begin{array}{c}\text { Rösch-MacAdam } \\
\text { Color Surface }\end{array}$ \\
\hline Normal & $2,094,542$ & 52,429 \\
\hline Protanomal & $1,433,194(68 \%)$ & $49,147(93 \%)$ \\
\hline Deuteranomal & $1,194,781(57 \%)$ & $48,001(91 \%)$ \\
\hline Tritanope & $17,626(0.84 \%)$ & $17,339(33 \%)$ \\
\hline Deuteranope & $16,036(0.76 \%)$ & $15,612(29 \%)$ \\
\hline Protanope & $16,093(0.76 \%)$ & $16,215(30 \%)$ \\
\hline
\end{tabular}

obtained for the Rösch-MacAdam color volume but similar to the one obtained across the Rösch-MacAdam color surface.

\section{DISCUSSION}

Estimating the number of discernible colors is a way to quantify chromatic discrimination over an extended region of color space. Although the absolute numbers obtained are informative and reflect the morphology of the space and the structure of the physical data under analysis, they are dependent on a number of assumptions. Relative numbers, however, are expected to be more robust and may provide more reliable information. Previous studies using this methodology have shown that only $30 \%$ of the theoretical maximum for the full object-color solid are actually occupied by colors found in nature [9], that in dichromatic vision the fraction of colors perceived in natural environments is about $7 \%$ of those perceived by normal color vision [10], and that the spectral structure of the illumination influences dramatically the number of colors perceived and hence color rendering [4,19,44]. These results represent fundamental information about the visual system and natural physical stimuli that would be difficult to derive in any other way. In the present work we show that the relative reduction in the number of discernible colors for the different types of color-deficient observers in relation to a color normal observer is an adequate way of expressing synthetically the complex relations among the corresponding color perceptions.

The impairment in chromatic discrimination for colordeficient observers was quantified by estimating the number of discernible colors perceived by each type of colordeficient observer. The set of the spectral reflectances defining the optimal colors was assumed to be illuminated by D65, and the object-color volumes for each observer were computed. These computations were carried out using models of color-deficient perception simulating for normal trichromatic observers the appearance of the corresponding colors for color-deficient observers. The extent of these volumes was then used as a measure of the number of discernible colors. It was found that dichromats perceive about $1 \%-0.5 \%$ of the colors perceived by normal observers, deuteranomals about 50\%-60\%. and prota- 

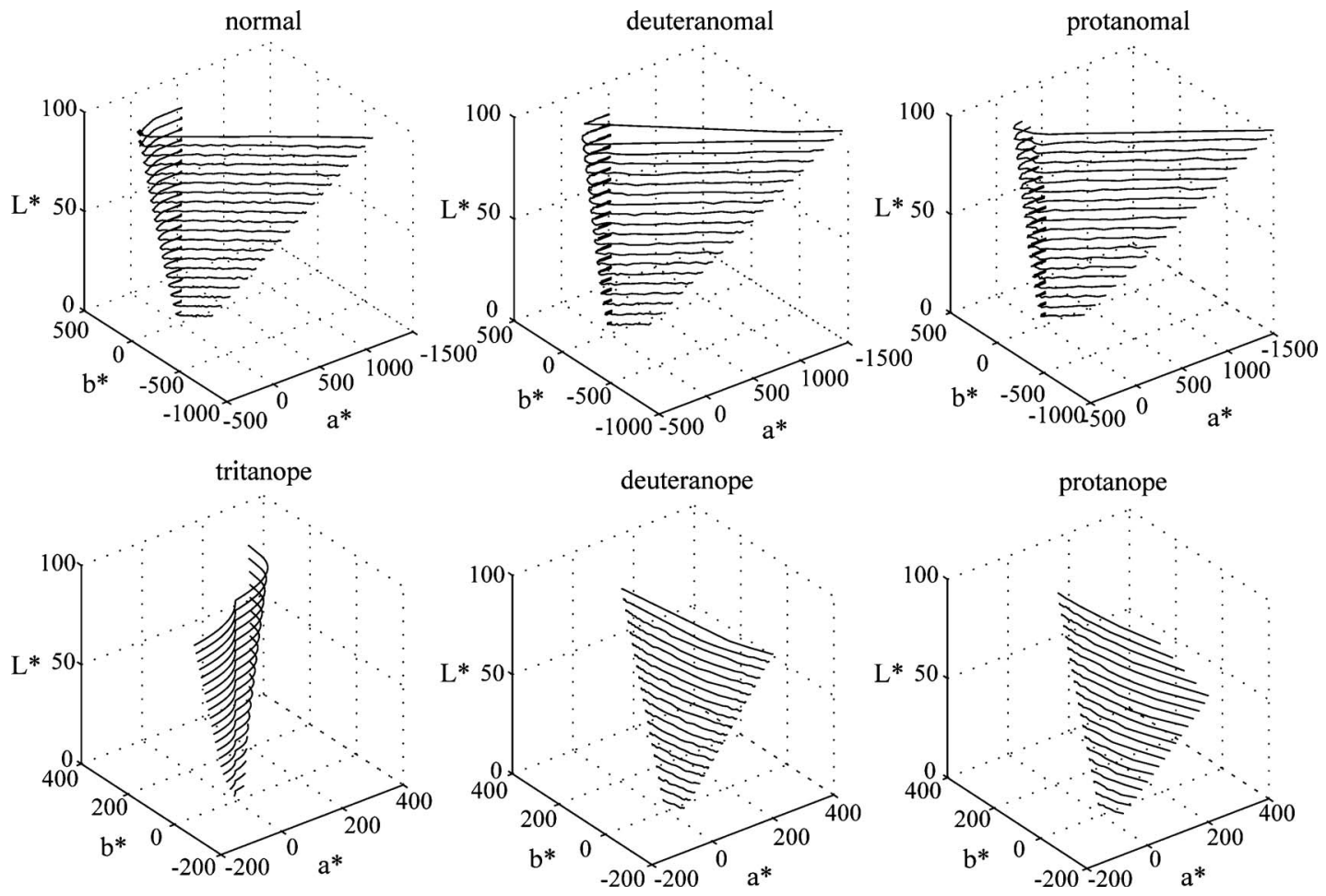

Fig. 4. Spectral locus of monochromatic stimuli represented in the CIELAB color space as perceived by normal, dicromats, and anomalous observers. The orientation of these graphs is different from those in Figs. 1 and 2 for better visualization.

nomals $60 \%-70 \%$. For anomalous trichromats, variations up to $40 \%$ around these values were found depending on the severity of the anomaly.

These estimates are independent of empirical measurements of the spectral reflectances and sampling and assume that all possible object colors occur in nature. Thus, they represent a theoretical limiting condition. Estimates derived from hyperspectral data of natural scenes suggest less impairment for dichromats [10] and for anomalous trichromats [18]. This apparent inconsistency arises because of the fact that in nature not all colors truly occur; that is, there are empty regions within the color volume.
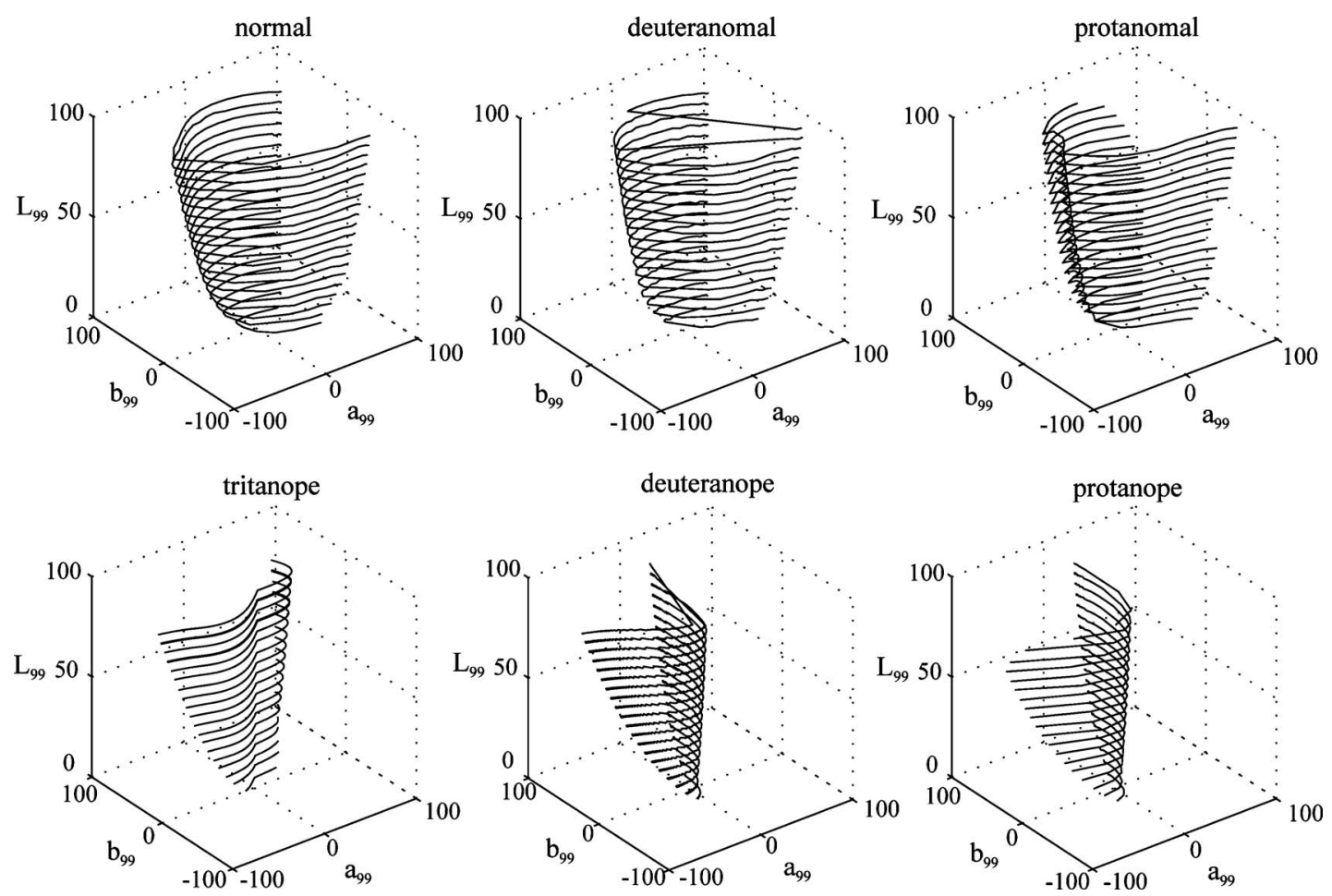

Fig. 5. Spectral locus of monochromatic stimuli represented in the DIN99d color space as perceived by normal, dicromats, and anomalous observers. The orientation of these graphs is different from those in Figs. 1 and 2 for better visualization. 


\section{Table 3. Number of Discernible Colors Estimated for the Spectral Locus of Monochromatic Stimuli Expressed in the CIELAB and DIN99d Color Spaces for Normal and Color-Deficient Observers}

Number of Discernible Colors Across the Spectral Locus of Monochromatic Stimuli

\begin{tabular}{lccc}
\hline & \multicolumn{1}{c}{ CIELAB } & & DIN99d \\
\cline { 2 - 2 } Normal & 179,541 & & 56,678 \\
Protanomal & $175,646(98 \%)$ & & $54,418(96 \%)$ \\
Deuteranomal & $167,940(94 \%)$ & & $47,765(84 \%)$ \\
Tritanope & $39,030(22 \%)$ & & $25,906(45 \%)$ \\
Deuteranope & $32,314(18 \%)$ & & $28,891(51 \%)$ \\
Protanope & $34,008(19 \%)$ & & $30,356(54 \%)$ \\
\hline
\end{tabular}

Estimation of the gamut of real colors can be obtained from sets of representative samples from industrial color atlases, colors of plants and vegetables, pigments from paints, and colors of textiles, among others. These colors represent about half of the theoretical maximum [45]. On the other hand, an estimate based on hyperspectral data from natural scenes suggests that only about $30 \%$ of the possible color volume is occupied [9]. In particular, in the specific condition of dichromacy, where the color volume is projected onto a surface, the fraction of colors perceived is expected to be smaller for the object-color solid than for natural scenes, because the volume of the former is larger than that of the latter and therefore its projection is proportionally smaller.

The estimated reduction in the number of colors was similar for the three types of dichromats; that is, the sacrifice of a cone class produced similar reduction regardless of the class. This result is consistent with previous estimates based on natural scenes, where it was found that for dichromatic vision the number of discernible colors was about $7 \%$ of normal trichromatic vision regardless of the cone class considered [10]. This is a consequence of the almost cylindrical symmetry of the Rösch-MacAdam color solid (see Figs. 1 and 2): the projection of the solid in a specific direction corresponding to each particular color deficiency is almost independent of the direction. This type of symmetry is also present on the colors from natural scenes as shown by Linhares et al. [9]. Note that this symmetry refers only to a chromatic gamut and not to a distribution of colors, which is of course not uniform across the gamut. Also, the symmetry is not observable when the data are represented on a cone space or chromaticity space: for the colors of natural scenes the redundancy between the $\mathrm{M}$ and $\mathrm{L}$ cones is clear when the data are represented in cone space [46], and a clear asymmetry is also evident when the data are represented in a chromaticity diagram $[47,48]$. This difference between physical and perceptual representations is probably a product of the decorrelation of cone signals, which has been shown to be efficiently encoded by opponent channels $[46,49,50]$. Thus, the symmetry of the effects of the lack of a cone type on the number of discernible colors makes evident an important property of the visual system.

Estimates of the reduction in chromatic discrimination perceived by color-deficient observers were obtained for dichromats using color naming along the monochromatic locus by estimating the reduction in transmitted information about the wavelength or intensity [51]. The data derived from four wavelengths suggest reductions in information transmitted from about $30 \%$ to about $1 \%$, depending on the observers and type of dichromacy. For the monochromatic stimuli and dichromatic observers, we obtain impairments of the order of $20 \%$ with CIELAB. Although, strictly, these numbers cannot be directly compared since they refer to different quantities, they give a notion of the order of magnitude of the impairment and are broadly consistent. But why is the impairment less severe for monochromatic stimuli than for the stimuli inside the color volume defined by the optimal colors? For the latter, color deficiencies imply a fractional reduction in the color volume; for the former, the reduction occurs in the area of a surface defined in color space. Because area and volume have different dimensionality, the estimates based on the volume are expected to be lower than those based on area.

The methodology to estimate the number of discernible colors is, of course, only approximate. On the one hand, the square packing method considers all colors within the same cube to be indistinguishable; segmenting the space with spheres would be a better approximation to guarantee that all color pairs are below the threshold. On the other hand, although the DIN99d color space is reasonably uniform, its non-uniformity in specific regions of the color space is well documented [52]. Also, these spaces are optimized for colored samples viewed in very specific conditions, and their performance in natural scenarios is unknown. Yet, these are probably the best estimations with present knowledge, and it is unlikely that correcting for these effects will dramatically change the main results derived here.

The model used to represent dichromatic vision has limitations [53], but it has been used to obtain approximated descriptions in real-world scenes [54] and in practical applications [55-57]. Other available models [53,58] are also limited and are not optimized to work with extreme chromatic ranges of the optimal colors; thus it is unclear whether they can be used advantageously in these types of analysis. In addition, the estimates apply to only small-field stimuli and do not take into account the well-documented improvement of color discrimination for large-field stimuli $[51,59,60]$.

The simulation of the color vision of anomalous trichromats was based on the assumption that the color vision system of these observers is in all ways equal to that of the normal observer except for the spectral sensitivities of the cone photoreceptors. Thus, any given trio of cone excitations produces the same color perception in the anomalous as in the color normal observer. Although it may be expected that color anomalous observers have different normalizations that imply that the above assumption does not hold, at present there is no available model that takes into account the normalization effects.

This analysis is based on the idealized theoretical limits and complements estimates based on empirical data that produce a quantitative limit of the chromatic impairment of color-deficient observers. 


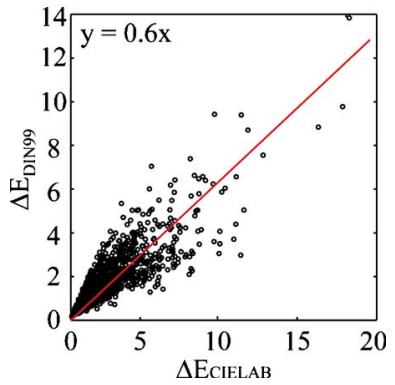

Fig. 6. (Color online) Color differences in DIN99d for the pairs BFD-P [61], RIT-DuPont [62], Leeds [63], and Witt [64] expressed as a function of the corresponding color differences expressed in the CIELAB color space.

\section{APPENDIX A: DISCRIMINATION THRESHOLD IN DIN99}

To estimate the relationship between discrimination thresholds in CIELAB and DIN99d, a color discrimination dataset with small-magnitude color differences (SCDs) was used. The set included four sub-datasets: BFD-P [61], RIT-DuPont [62], Leeds [63], and Witt [64], with $2776,156,307$, and 418 pairs, respectively, and an average of $2.5 \Delta \mathrm{E}^{*}{ }_{a b}$ [52]. A weighting factor was applied to each individual dataset to compensate for the relative differences in number of samples. The color differences were computed for each pair assumed to be illuminated under D65 in the two color spaces. Figure 6 represents $\Delta \mathrm{E}_{\text {DIN99d }}$ as a function of $\Delta \mathrm{E}_{\text {CIELAB }}$ for some of the pairs in the sample used. A linear fit to the data gives a slope of 0.6 , and this was the value used for discrimination threshold in DIN99d.

\section{ACKNOWLEDGMENTS}

This work was supported by the Spanish Ministry for Education and Science (DPI2005-08999-C02-02); the Spanish Ministry of Science and Innovation (DPI200806455-C02-02); the Centro de Física at Minho University, Braga, Portugal and the Portuguese Fundação para a Ciência e a Tecnologia (PTDC/EEA-EEL/098572/2008). Esther Perales was supported by the Spanish Ministry for Education and Science with the BES-2006-13518 grant, and João M. M. Linhares was supported by the Portuguese Fundação para a Ciência e a Tecnologia with the SFRH/BD/35874/ grant. We are grateful to David H. Foster for helpful discussions and to two reviewers and the editor for valuable advice.

\section{REFERENCES}

1. L. T. Sharpe, A. Stockman, H. Jagle, and J. Nathans, "Opsin genes, cone photopigments, color vision, and color blindness," in Color Vision, K. R. Gegenfurtner and L. T. Sharpe, eds. (Cambridge University Press, 1999), pp. 3-51.

2. S. G. Solomon and P. Lennie, "The machinery of colour vision," Nat. Rev. Neurosci. 8, 276-286 (2007).

3. D. L. MacAdam, "Note on the number of distinct chromaticities," J. Opt. Soc. Am. 37, 308-309 (1947).

4. F. Martinez-Verdu, E. Perales, E. Chorro, D. de Fez, V. Viqueira, and E. Gilabert, "Computation and visualization of the MacAdam limits for any lightness, hue angle, and light source," J. Opt. Soc. Am. A 24, 1501-1515 (2007).

5. E. Perales, F. Martinez-Verdu, V. Viqueira, M. J. Luque, and P. Capilla, "Computing the number of distinguishable colors under several illuminants and light sources," in Third IS\&T European Conferences on Colour Graphics, Imaging and Vision (Society for Imaging Science and Technology, 2006), pp. 414-419.

6. M. R. Pointer, "On the number of discernible colours," Color Res. Appl. 23, 337 (1998).

7. M. R. Pointer and G. G. Attridge, "The number of discernible colours," Color Res. Appl. 23, 52-54 (1998).

8. E. Perales, "About the optimal colours and their applications in colour vision and colour technology," Ph.D. thesis (University of Alicante, 2009).

9. J. M. Linhares, P. D. Pinto, and S. M. Nascimento, "The number of discernible colors in natural scenes," J. Opt. Soc. Am. A 25, 2918-2924 (2008).

10. J. M. M. Linhares, P. D. Pinto, and S. M. C. Nascimento, "The number of discernible colors perceived by dichromats in natural scenes and the effects of colored lenses," Visual Neurosci. 25, 493-499 (2008).

11. I. Marín-Franch and D. H. Foster, "Number of perceptually distinct surface colors in natural scenes," J. Vision (2010) (to be published).

12. M. Neitz and J. Neitz, "Molecular genetics of color vision and color vision defects," Arch. Ophthalmol. (Chicago) 118, 691-700 (2000).

13. J. Pokorny, V. C. Smith, and G. Verriest, "Congenital color defects," in Congenital and Acquired Color Vision Defects, J. Pokorny, V. C. Smith, G. Verriest, and A. J. L. G. Pinckers, eds. (Grune and Stratton, New York, 1979), pp. 183-241.

14. J. Birch, J. A. Chisholm, P. Kinnea, M. Marre, A. J. L. G. Pinckers, J. Pokorny, V. C. Smith, and G. Verriest, "Acquired color vision defects," in Congenital and Acquired Color Vision Defects, J. Pokorny, V. C. Smith, G. Verriest, and A. J. L. G. Pinckers, eds. (Grune \& Stratton, 1979), pp. 243-348.

15. H. Krastel and J. D. Moreland, "Colour vision deficiencies in ophthalmic diseases," in Inherited and Acquired Colour Vision Deficiencies: Fundamental Aspects and Clinical Studie, D. H. Foster, ed. (Macmillan, 1991), pp. 115-172.

16. M. J. Morgan, A. Adam, and J. D. Mollon, "Dichromates detect color-camouflaged objects that are not detected by trichromates," Proc. R. Soc. London Ser. B 248, 291-295 (1992).

17. L. T. Sharpe, E. de Luca, T. Hansen, H. Jagle, and K. R. Gegenfurtner, "Advantages and disadvantages of human dichromacy," J. Vision 6, 213-223 (2006).

18. J. M. M. Linhares, P. A. Pinto, and S. M. C. Nascimento, "The number of discernible colours perceived by protanomalous and deuteranomalous in natural scenes," Perception 37, 62-62 (2008).

19. J. M. Linhares, P. D. Pinto, and S. M. Nascimento, "Color rendering of art paintings under CIE illuminants for normal and color-deficient observers," J. Opt. Soc. Am. A 26, 1668-1677 (2009).

20. G. Wyszecki and W. S. Stiles, Color Science: Concepts and Methods, Quantitative Data and Formulae, 2nd ed. (Wiley, New York, 1982).

21. E. Schrodinger, "Theorie der pigmente von grosster leuchtkraft," Annalen Physik 62, 603-622 (1920).

22. R. Luther, "Aus dem Gebiet der Farbreizmetrik," Z. Tech. Phys. (Leipzig) 8, 540-558 (1927).

23. N. D. Nyberg, "Zum Aufbau des Farbenkörpers im Raume aller Lichtempfindungen,” Z. Phys. A 52, 406-419 (1929).

24. D. L. MacAdam, "Theory of the maximum visual efficiency of colored materials," J. Opt. Soc. Am. 25, 249-252 (1935).

25. D. L. MacAdam, "Maximum visual efficiency of colored materials," J. Opt. Soc. Am. 25, 316-367 (1935).

26. J. von Kries, "Die Gesichtsempfindungen und ihre Analyse," Archiv fur Anatomie, Physiologie und wissenschaftliche Medicin (Suppl. Physiologische Abteilung), 1-178 (1882).

27. D. B. Judd and K. L. Kelly, "Method of designating colors," J. Res. Natl. Bur. Stand. 23, 355-381 (1939).

28. J. Krauskopf and K. Gegenfurtner, "Color discrimination and adaptation," Vision Res. 32, 2165-2175 (1992).

29. S. Wen, "Display gamut comparison with number of dis- 
cernible colors," J. Electron. Imaging 15, 043001-043008 (2006).

30. H. Xu, "Color-rendering capacity of light," Color Res. Appl. 18, 267-269 (1993).

31. CIE, "Colorimetry," CIE Publ 15:2004 (CIE, Vienna, 2004).

32. G. Cui, M. R. Luo, B. Rigg, G. Roesler, and K. Witt, "Uniform colour spaces based on the DIN99 colour-difference formula," Color Res. Appl. 27, 282-290 (2002).

33. R. G. Kuehni, Color Space and Its Divisions: Color Order from Antiquity to the Present (Wiley-Interscience, 2003).

34. H. Brettel, F. Viénot, and J. D. Mollon, "Computerized simulation of color appearance for dichromats," J. Opt. Soc. Am. A 14, 2647-2655 (1997).

35. F. Vienot, H. Brettel, L. Ott, A. Benmbarek, and J. D. Mollon, "What do color-blind people see?" Nature 376, 127-128 (1995).

36. D. B. Judd, "Color perceptions of deuteranopic and protanopic observers," J. Res. Natl. Bur. Stand. 41, 247-271 (1949).

37. V. C. Smith and J. Pokorny, "Spectral sensitivity of colorblind observers and the cone photopigments," Vision Res. 12, 2059-2071 (1972).

38. J. J. Vos, "Colorimetric and photometric properties of a $2^{\circ}$ fundamental observer," Color Res. Appl. 3, 125-128 (1978).

39. P. DeMarco, J. Pokorny, and V. C. Smith, "Full-spectrum cone sensitivity functions for X-chromosome-linked anomalous trichromates," J. Opt. Soc. Am. A 9, 1465-1476 (1992).

40. A. Knowles and H. J. A. Dartnall, "The photobiology of vision," in The Eye, Vol. 2B, H. Davson, ed. (Academic, 1997), pp. $53-101$.

41. S. L. Merbs and J. Nathans, "Absorption-spectra of the hybrid pigments responsible for anomalous color-vision," Science 258, 464-466 (1992).

42. E. Perales, F. Martínez-Verdú, V. Viqueira, M. J. Luque, and P. Capilla, "Computing the number of distinguishable colors under several illuminants and light sources," in Third IS\&T European Conferences on Colour Graphics, Imaging and Vision (The Society for Imaging Science and Technology, 2006), pp. 414-419.

43. N. Moroney, "A hypothesis regarding the poor blue constancy of CIELAB," Color Res. Appl. 28, 371-378 (2002).

44. P. D. Pinto, J. M. M. Linhares, and S. M. C. Nascimento, "Correlated color temperature preferred by observers for illumination of artistic paintings," J. Opt. Soc. Am. A 25, 623-630 (2008).

45. E. Perales, E. Chorro, V. Viqueira, and F. M. MartínezVerdú, "Estimation of the real colour gamut," in 11th Congress of the International Colour Association (AIC 2009) (Colour Society of Australia, 2009), p. 71.

46. D. L. Ruderman, T. W. Cronin, and C. C. Chiao, "Statistics of cone responses to natural images: implications for visual coding," J. Opt. Soc. Am. A 15, 2036-2045 (1998).
47. M. A. Webster and J. D. Mollon, "Adaptation and the color statistics of natural images," Vision Res. 37, 3283-3298 (1997).

48. S. M. C. Nascimento, F. P. Ferreira, and D. H. Foster, "Statistics of spatial cone-excitation ratios in natural scenes," J. Opt. Soc. Am. A 19, 1484-1490 (2002).

49. G. Buchsbaum and A. Gottschalk, "Trichromacy, opponent colors coding and optimum color information-transmission in the retina," Proc. R. Soc. London Ser. B 220, 89-113 (1983).

50. Q. Zaidi, "Decorrelation of L- and M-cone signals," J. Opt. Soc. Am. A 14, 3430-3431 (1997).

51. A. L. Nagy and R. M. Boynton, "Large-field color naming of dichromates with rods bleached," J. Opt. Soc. Am. 69, 12591265 (1979).

52. M. R. Luo, G. Cui, and C. Li, "Uniform colour spaces based on CIECAM02 colour appearance model," Color Res. Appl. 31, 320-330 (2006).

53. T. Wachtler, U. Dohrmann, and R. Hertel, "Modeling color percepts of dichromats," Vision Res. 44, 2843-2855 (2004).

54. J. D. Mollon and B. C. Regan, "Simulating the perceptual world of the anomalous trichromat," Invest. Ophthalmol. Visual Sci. 42, S97-S97 (2001).

55. V. A. Kovalev, "Mining dichromatic colours from video," Lect. Notes Artif. Int. 4065, 431-443 (2006).

56. K. Rasche, R. Geist, and J. Westall, "Detail preserving reproduction of color images for monochromats and dichromats," IEEE Comput. Graphics Appl. 25, 22-30 (2005).

57. F. Vienot, H. Brettel, and J. D. Mollon, "Digital video colourmaps for checking the legibility of displays by dichromats," Color Res. Appl. 24, 243-252 (1999).

58. P. Capilla, M. J. Luque, and M. A. Diez-Ajenjo, "Looking for the dichromatic version of a colour vision model," J. Opt. A, Pure Appl. Opt. 6, 906-919 (2004).

59. A. L. Nagy, "Large-field substitution Rayleigh matches of dichromats," J. Opt. Soc. Am. 70, 778-784 (1980).

60. V. C. Smith and J. Pokorny, "Large-field trichromacy in protanopes and deuteranopes," J. Opt. Soc. Am. 67, 213-220 (1977).

61. M. R. Luo and B. Rigg, "Chromaticity-discrimination ellipses for surface colours," Color Res. Appl. 11, 25-42 (1986).

62. R. S. Berns, D. H. Alman, L. Reniff, G. D. Snyder, and M. R. Balonon-Rosen, "Visual determination of suprathreshold colour-difference tolerances using probit analysis," Color Res. Appl. 16, 297-316 (1991).

63. D. H. Kim and J. H. Nobbs, "New weighting functions for the weighted CIELAB colour-difference formula," in 8th Congress of the International Colour Association (AIC 97) (Color Association of China, 1997), pp. 446-449.

64. K. Witt, "Geometric relations between scales of small colour-differences," Color Res. Appl. 24, 78-92 (1999). 